Bull. Austral. Math. Soc.

$11 \mathrm{~J} 68,11 \mathrm{~N} 25$

VoL. $71(2005)$ [11-16]

\title{
POWERFUL NUMBERS IN SHORT INTERVALS
}

\author{
Jean-Marie De Koninck, Florian Luca and Igor E. ShParlinski
}

Let $\kappa \geqslant 2$ be an integer. We show that there exist infinitely many positive integers $N$ such that the number of $\kappa$-full integers in the interval $\left(N^{\kappa},(N+1)^{\kappa}\right)$ is at least $(\log N)^{1 / 3+o(1)}$. We also show that the $A B C$-conjecture implies that for any fixed $\delta>0$ and sufficiently large $N$, the interval $\left(N, N+N^{1-(2+\delta) / \kappa}\right)$ contains at most one $\kappa$-full number.

\section{INTRODUCTION}

Let $\kappa>1$ be an integer. An integer $m \geqslant 1$ is called $\kappa$-full if $p^{\kappa} \mid m$ holds for all prime factors $p$ of $m$. For example, $\kappa$-powers, that is, numbers $m$ of the form $n^{\kappa}$, are $\kappa$-full. Usually, for $\kappa=2$ such numbers are called squarefull.

It is clear that for any integer $N$, the open interval $\left(N^{\kappa},(N+1)^{\kappa}\right)$ does not contain any $\kappa$-powers. In this paper, we show that the intervals of the above form can contain arbitrarily many $\kappa$-full numbers. This result extends some of the results obtained in [3], where squarefull numbers have been investigated. It is useful to recall that the counting function of the $\kappa$-full numbers up to $x$ is of the same order of magnitude as the counting function of the $\kappa$-powers (see [1]), thus this difference in their behaviour is based on purely arithmetic reasons.

The proof is an extension of that given in [3] but uses the Roth Theorem instead of a result on continued fractions of some quadratic irrationalities. Alternatively, one can use the fully effective Liouville Theorem (which leads to a marginally weaker but more uniform statement). In fact, even in the case $\kappa=2$ both these approaches lead to a slightly better constant than that of [3].

We recall that the Roth Theorem asserts that for any irrational root $\alpha$ of a monic irreducible polynomial $f(X) \in \mathbb{Q}[X]$ and any $\delta>0$, there exists a constant $C(\alpha, \delta)>0$ such that for any integers $r$ and $s>0$, we have

$$
\left|\alpha-\frac{r}{s}\right|>\frac{C(\alpha, \delta)}{s^{2+\delta}}
$$

Received 10th May, 2004

During the preparation of this paper, the first author was supported in part by a grant from NSERC. The second author was supported in part by grants SEP-CONACYT 37259-E and 37260-E, and the third author was supported in part by ARC grant DP0211459.

Copyright Clearance Centre, Inc. Serial-fee code: 0004-9727/05 \$A2.00+0.00. 
However, this result is not effective in a sense that no explicit expression for $C(\alpha, \delta)$ is known (see [5, Theorem 2A of Chapter 5]).

We also recall the Dirichlet Theorem which asserts that, for any real numbers $\alpha_{1}, \ldots, \alpha_{m}$ and integer $Q>1$, there exist integers $r_{1}, \ldots, r_{m}$ and $0<s \leqslant Q$ such that

$$
\left|\alpha_{j}-\frac{r_{j}}{s}\right| \leqslant \frac{1}{s Q^{1 / m}}
$$

(see [5, Theorem 1A of Chapter 2]).

We also show that the $A B C$-conjecture implies that for any $\delta>0$ and sufficiently large $L$, the shorter interval $\left(L, L+L^{1-(2+\delta) / \kappa}\right)$ contains at most one $\kappa$-full number.

We also obtain an unconditional (but much weaker) upper bound on the number of integers in short intervals $(L, L+K)$, which are $\kappa$-full numbers for at least one $\kappa \geqslant 2$. This complements the result obtained in [4], where the upper bound $\exp \left(40(\log \log L \log \log \log L)^{1 / 2}\right)$ on the number of perfect powers in the interval $(L, L$ $+L^{1 / 2}$ ) (provided $L \geqslant 16$ ) is established.

Throughout this paper, we use Vinogradov symbols $\gg$ and $\ll$ as well as Landau symbols $O$ and $o$ with their regular meanings. We recall that the notations $A \ll B$, $B \gg A$ and $A=O(B)$ are all equivalent to the fact that $|A| \leqslant c B$ holds with positive constant $c$.

\section{Short Intervals With MaNy $\kappa$-Full Numbers}

THEOREM 1. For any integer $\kappa \geqslant 2$, there are infinitely many $N$, such that the open interval $\left(N^{\kappa},(N+1)^{\kappa}\right)$ contains at least

$$
M \geqslant\left(\left(\frac{3}{8}+o(1)\right) \frac{\log N}{\log \log N}\right)^{1 / 3}
$$

$\kappa$-full integers.

Proof: Let $1<d_{1}<\ldots<d_{2 \ell}$ be the first $2 \ell$ squarefree integers greater than 1 , that is, $d_{j}=\pi^{2} j / 6+o(j)$. We also denote

$$
D=\prod_{j=1}^{2 \ell} d_{j}
$$

and remark that $D \leqslant(4 \ell)^{2 \ell}$, provided that $\ell$ is large enough.

Let $\alpha_{j}=d_{j}^{-1 / \kappa}, j=1, \ldots, 2 \ell$.

We define

$$
R=\left(\kappa 2^{\kappa-1}(4 \ell)^{2 \ell+1 / \kappa}\right)^{2 \ell}
$$

and let $q$ be the smallest integer

$$
q \geqslant R
$$


for which, for some integers $r_{j}$,

$$
\left|\alpha_{j}-\frac{r_{j}}{q}\right| \leqslant \frac{1}{q^{1+1 / 2 \ell}}, \quad j=1, \ldots, 2 \ell .
$$

We see that

$$
q \leqslant Q,
$$

where $Q=R^{2 \ell(1+\delta)} C\left(\alpha_{1}, \delta\right)^{-2 \ell}$. Indeed, otherwise applying the Dirichlet Theorem, we see that

$$
\left|\alpha_{j}-\frac{r_{j}}{s}\right| \leqslant \frac{1}{s Q^{1 / 2 \ell}}, \quad j=1, \ldots, 2 \ell,
$$

for some positive integer $s \leqslant Q$. Due to the minimality condition on $q$, we have $s \leqslant R$. On the other hand, by the Roth Theorem, we have

$$
\frac{C\left(\alpha_{1}, \delta\right)}{s^{2+\delta}}<\left|\alpha_{1}-\frac{r_{1}}{s}\right| \leqslant \frac{1}{s Q^{1 / 2 \ell}}
$$

Therefore

$$
s>\left(C\left(\alpha_{1}, \delta\right) Q^{1 / 2 \ell}\right)^{1 /(1+\delta)}=R,
$$

which is impossible.

We see from (1) that, for $j=1, \ldots, 2 \ell$,

$$
\left|q-d_{j}^{1 / \kappa} r_{j}\right| \leqslant \frac{d_{j}^{1 / \kappa}}{q^{1 / 2 \ell}} \leqslant \frac{(4 \ell)^{1 / \kappa}}{R^{1 / 2 \ell}} \leqslant 1
$$

Therefore,

$$
d_{j}^{1 / \kappa} r_{j}=\alpha_{j}^{-1} r_{j} \leqslant q+1, \quad j=1, \ldots, 2 \ell
$$

We now derive,

$$
\begin{aligned}
\left|q^{\kappa}-d_{j} r_{j}^{\kappa}\right| & =\left|q-d_{j}^{1 / \kappa} r_{j}\right| \sum_{\nu=0}^{\kappa-1} q^{\kappa-1-\nu}\left(d_{j} r_{j}\right)^{\nu} \leqslant \kappa(q+1)^{\kappa-1}\left|q-d_{j}^{1 / \kappa} r_{j}\right| \\
& \leqslant \frac{\kappa(4 \ell)^{1 / \kappa}(q+1)^{\kappa-1}}{R^{1 / 2 \ell}} .
\end{aligned}
$$

Putting $n=D q$ we derive

$$
\begin{aligned}
\left|n^{\kappa}-d_{j} D^{\kappa} r_{j}^{\kappa}\right| & \leqslant \frac{\kappa(4 \ell)^{1 / \kappa} D^{\kappa}(q+1)^{\kappa-1}}{R^{1 / 2 \ell}} \\
& \leqslant D^{\kappa-1} q^{\kappa-1} \frac{\kappa(4 \ell)^{2 \ell+1 / \kappa}(1+1 / q)^{\kappa-1}}{R^{1 / 2 \ell}} \\
& =n^{\kappa-1} \frac{\kappa(4 \ell)^{2 \ell+1 / \kappa}(1+1 / q)^{\kappa-1}}{R^{1 / 2 \ell}} \\
& \leqslant n^{\kappa-1} \frac{\kappa 2^{\kappa-1}(4 \ell)^{2 \ell+1 / \kappa}}{R^{1 / 2 \ell}}=n^{\kappa-1} .
\end{aligned}
$$


Therefore one of the intervals $\left((n-1)^{\kappa}, n^{\kappa}\right)$ or $\left(n^{\kappa},(n+1)^{\kappa}\right)$ contains at least $M \geqslant \ell$ of the integers $d_{j} D^{\kappa} r_{j}^{\kappa}, j=1, \ldots, 2 \ell$, which are obviously pairwise distinct (because $d_{j}$ is squarefree for all $j=1, \ldots, 2 \ell$ ), and $\kappa$-full. We now have

$$
\begin{aligned}
n & =D q \leqslant D Q=D R^{2 \ell(1+\delta)} C\left(\alpha_{1}, \delta\right)^{-2 \ell} \\
& \leqslant\left(4 \ell / C\left(\alpha_{1}, \delta\right)\right)^{2 \ell}\left(\kappa 2^{\kappa-1}(4 \ell)^{2 \ell+1 / \kappa}\right)^{4 \ell^{2}(1+\delta)} \\
& =\exp \left((8(1+\delta)+o(1)) \ell^{3} \log \ell\right) .
\end{aligned}
$$

Hence, since $\kappa$ is fixed,

$$
\ell^{3} \log \ell \geqslant\left(\frac{1}{8(1+\delta)}+o(1)\right) \log n
$$

which implies that

$$
M \geqslant\left(\left(\frac{3}{8(1+\delta)}\right)^{1 / 3}+o(1)\right)\left(\frac{\log n}{\log \log n}\right)^{1 / 3}
$$

Recalling that $\delta$ is arbitrary, the proof is complete.

\section{UPPER BOUNDS}

We first recall the statement of the $A B C$-conjecture. For any nonzero integer $m$ let

$$
\gamma(m)=\prod_{p \mid m} p
$$

CONJECTURE 1. For every $\varepsilon>0$ there exists a constant $C(\varepsilon)$ such that for any integers $a, b, c$ with $c=a+b$ and $\operatorname{gcd}(a, b)=1$, the bound

$$
\max \{|a|,|b|,|c|\} \leqslant C(\varepsilon) \gamma(a b c)^{1+\varepsilon}
$$

holds.

THEOREM 2. The $A B C$-conjecture implies that if $\kappa$ and $\delta>0$ are fixed, then there exists $L_{0}$ such that the interval $\left(L, L+L^{1-(2+\delta) / \kappa}\right)$ contains at most one $\kappa$-full number for $L>L_{0}$.

Proof: Let $\varepsilon=\delta / \kappa$. Assume that the above interval contains at least two $\kappa$-full numbers, say $a<b$. Then $\gamma(a), \gamma(b) \leqslant(2 L)^{1 / \kappa}$ and $c=b-a<L^{1-(2+\delta) / \kappa}$. Applying the $A B C$-conjecture to the equation $c=b-a$, we get

$$
L<b \leqslant C(\varepsilon)\left(2^{2 / \kappa} L^{1-\delta / \kappa}\right)^{1+\varepsilon}=C(\delta / \kappa) 2^{2 / \kappa+2 \delta / \kappa^{2}} L^{1-\delta^{2} / \kappa^{2}} .
$$

Hence

$$
L<\left(C(\delta / \kappa) 2^{2 / \kappa+2 \delta / \kappa^{2}}\right)^{\kappa^{2} / \delta^{2}}
$$


which completes the proof.

We remark that, unfortunately, the best known results towards the $A B C$-conjecture (see [6]), are not strong enough to produce any nontrivial estimates for $\kappa$-full numbers in short intervals.

We now obtain a much weaker but unconditional bound.

THEOREM 3. For any positive integers $L$ and $K$ the interval $(L, L+K)$ contains at most $O(K \log \log K / \log K)$ squarefull numbers.

Proof: Let

$$
w=\frac{\log K}{\log \log K}
$$

We separate the squarefull numbers of the interval $(L, L+K)$ into two nonintersecting subsets. The set $\mathcal{S}_{1}$ consists of the squarefull numbers which have a prime divisor $p$ with $w \leqslant p \leqslant K$ (and thus are divisible by $p^{2}$ ). The set $\mathcal{S}_{2}$ consists of all other squarefull numbers in this interval. Clearly,

$$
\# \mathcal{S}_{1} \leqslant \sum_{w \leqslant p<K}\left(\frac{K}{p^{2}}+1\right) \ll \frac{K}{w \log w}+\frac{K}{\log K} \ll \frac{K}{\log K} .
$$

Using the Brun sieve (see [2, Theorem 2.2]), we also derive

$$
\# \mathcal{S}_{2} \ll K \prod_{w \leqslant p \leqslant K}\left(1-\frac{1}{p}\right) \ll \frac{K \log \log K}{\log K},
$$

which completes the proof.

\section{REMARKS}

As we have mentioned, the Roth Theorem is not effective. However, our arguments can be used with the completely explicit Liouville Theorem which asserts that for any irrational root $\alpha$ of a monic irreducible polynomial $f(X) \in \mathbb{Q}[X]$ of degree $\operatorname{deg} f=k \geqslant 2$ and any integers $r$ and $s>0$,

$$
\left|\alpha-\frac{r}{s}\right| \geqslant \frac{c(\alpha)}{s^{k}}
$$

where $c(\alpha)>0$ depends only on $\alpha$ (see [5, Theorem 1A of Chapter 5]). It is easy to see from any standard proof of this inequality that the constant $c(\alpha)$ can be taken to be

$$
c(\alpha)=\left(\Delta \max _{t \in[\alpha-1, \alpha+1]}\left\{1,\left|f^{\prime}(t)\right|\right\}\right)^{-1},
$$

where $\Delta$ is the least common multiple of all the denominators of the coefficients of $f$. For example, when $f(X)=X^{\kappa}-1 / d$ with some positive integers $\kappa \geqslant 2$ and $d$ and $\alpha=d^{-1 / \kappa}$, we can take $c(\alpha)=1 /\left(d \kappa 2^{\kappa-1}\right)$. 
Using this result leads to a uniform and explicit version of Theorem 1 with respect to $\kappa$ (the constant $(3 / 8)^{1 / 3}$ becomes $(3 / 8(\kappa-1))^{1 / 3}$ ). In particular, there are infinitely many $N$, such that the number of $\kappa(N)$-full integers in $\left(N^{\kappa(N)},(N+1)^{\kappa(N)}\right)$ is at least $(\log N)^{1 / 3+o(1)}$, where $\kappa(N)$ is any function of $N$ satisfying $\kappa(N)=(\log N)^{o(1)}$, for example. Moreover, the above interval can contain arbitrarily many $\kappa(N)$-full integers, where $\kappa(N)=o\left((\log N)^{1 / 2}\right)$.

\section{REFERENCES}

[1] P. Bateman and E. Grosswald, 'On a theorem of Erdös and Szekeres', Mlinois J. Math. 2 (1958), 88-98.

[2] H. Halberstam and H.-E. Richert, Sieve methods (Academic Press, London, 1974).

[3] J.-M. De Koninck and F. Luca, 'Sur la proximité des nombres puissants', Acta Arith. 114 (2004), 149-157.

[4] J.H. Loxton, 'Some problems involving powers of integers', Acta Arith. 46 (1986), 113-123.

[5] W.M. Schmidt, Diophantine approximation (Springer-Verlag, Berlin, 1980).

[6] C.L. Stewart and K.R. Yu, 'On the abc conjecture, II', Duke Math. J. 108 (2001), 169-181.

Départment de Mathématiques

Université Laval

Québec G1K 7P4

Canada

e-mail: jmdk@mat.ulaval.ca
Instituto de Matemáticas

Universidad Nacional Autónoma de México

C.P. 58180, Morelia

Michoacán

México

e-mail: fluca@matmor.unam.mx

Department of Computing

Macquarie University

Sydney, NSW 2109

Australia

e-mail: igor@ics.mq.edu.au 